\title{
STATUS OF CONVERSION OF DOE STANDARDS TO
} NON-GOVERNMENT STANDARDS

\section{Performance Assurance Project Office}

\author{
H. L. Moseley
}

Date Published - July 1992

Prepared for the

Department of Energy, Nuclear Energy KK 0502206

Prepared by the

OAK RIDGE NATIONAL LABORATORY

Oak Ridge, Tennessee 37831

managed by

M广 RTIN MAARIETTA ENERGY SYSTEMS, INC.

for the

DEPARTMENT OF ENERGY

under contract No. DE-AC05-840R21400 
CONTENTS

Page

FOREWORD $\ldots \ldots \ldots \ldots \ldots \ldots \ldots \ldots \ldots \ldots \ldots \ldots \ldots \ldots$

1. INTRODUCTION ......................... 1

2. CONVERSION PROCESS $\ldots \ldots \ldots \ldots \ldots \ldots \ldots \ldots \ldots \ldots \ldots \ldots \ldots$

2.1 Criteria to Identify Standards Eligible for Conversion ............ 2

2.2 Preparation of Plans to Implement Conversion $\ldots \ldots \ldots \ldots \ldots$

2.3 Development of a System to Monitor Conversion Activities . . . . . . . . 3

3. STATUS OF CONVERSION OF DOE STANDARDS TO

NON-GOVERNMENT STANDARDS $\ldots \ldots \ldots \ldots \ldots \ldots \ldots \ldots \ldots$ 


\section{FOREWORD}

One major goal of the Department of Energy (DOE) Technical Standards Program is to utilize a proven method for transferring DOE's program experience and technology to the private sector for commercial use. The proven method utilized by the Performance Assurance Project Office (Lead Standardization A stivity for DOE) is the "conversion" of DOE standards to non-Government standards (NGSs) when practicable. This method meets the policy and guidance established in OMB Circular No. A-119, Federal Participation in the Development and Use of Voluntary Standards, October 27, 1982 and the objectives of DOE Order 1300.2A, Department of Energy Technical Standards Program, May 19, 1992.

DOE standards were and are specifically designed to meet the needs of certain Department facilities, programs, and projects and, thus, are based on the highest level of DOE expertise and experience available at the time tile standards are developed. Modifications to the standards are made as experience is gained within DOE through application of the standards. Often, experience in the private sector provides the incentive to incorporate some or all requiremunts of specific Department standards into NGSs. This is the process referred to as "conversion." 


\section{INTRODUCTION}

One major goal of the DOE Technical Standards Program is to convert existing DOE standards into NGSs, where possible. This means that a DOE standard may form the basis for a standards-writing committee to produce a standard in the same subject area using the nonGovernment standards consensus process. This report is a summary of the activities that have evolved to effect conversion of DOE standards to NGSs, and the status of current conversion activities. In some cases, all requirements in a DOE standard will not be incorporated into the published non-Government standard because these requirements may be considered too restrictive or too specific for broader applicetion by private industry. If requirements in a DOE standard are not incorporated in a non-Government standard and the requirements are considered necessary for DOE program applications, the DOE standard will be revised and issued as a supplement to the non-Government standard. The DOE standard will contain only those necessary requirements not reflected by the non-Government standard. Therefore, while complete conversion of DOE standards may not always be realized, the Department's technical standards policy as stated in Order 1300.2A has been fully supported in attempting to make maximum use of the rion-Government standard.

\section{CONVERSION PROCESS}

All conversion efforts undertaken to date by DOE as detailed in this report involve standards developed under the DOE Nuclear Energy (NE) Standards Program. The NE Working Group on Standards developed three basic steps in managing the conversion effort.

1. Establish criteria to identify NE standards eligible for conversion.

2. Prepare action plans to implement conversion.

3. Develop a system to monitor conversion activities. 


\subsection{CRITERIA TO IDENTIFY STANDARDS ELIGIBLE FOR CONVERSION}

Early in the effort to establish criteria for standards to be converted, it was recognized that the need for a non-Government standard in a particular subject area should be the determining factor in evaluating the need to pursue conversion of an existing NE standard. Focusing on the aspect of need, the following criteria were established.

1. Current need

2. Future need

3. Past use

4. Scope and applicability

5. Special requirements

6. Quality assurance requirements
Is there a current (and continuing) need for the standard?

Is significant future application of the standard by private industry anticipated?

Does experience gained from previous application of the standard demonstrate a need for a nonGovernment standard?

Can the scope of applicability be converted to the needs of private industry?

Is there validity and justification for the requirements in private industry application?

For example, can ASME NOA-1 be applied instead of NE F 2-2 or NE F 2-4?

The criteria was applied in two steps.

1. If an individual NE standard met either criterion 1,2, or 3, the need for the NE standard was established.

2. If the NE standard then met criteria 4, 5, and 6, the NE standard should be selected as a candidate for conversion to a non-Government standard. 
Any DOE standard that is requested by a non-Government standards body (NGSB) for use in developing a non-Government standard will be made available to the requesting organization. The status of that effort will be included in this report, Status of Conversion of DOE Standards to Non-Government Standards, which is to be issued periodically by the Performance Assurance Project Office, (PAPO) as the Lead Standardization Activity for DOE.

\subsection{PREPARATION OF PLANS TO IMPLEMENT CONVERSION}

When a DOE standard is identified as a candidate for conversion to a non-Government standard, PAPO solicits information (from non-Government standards bodies and standards listings/data bases) on non-Government standards that appear to be similar in nature or scope to the DOE standard. Based on the information obtained, PAPO develops a plan for conversion of the DOE standard. Such a plan generally identified the non-Government standards body to be contacted, recommends an approach to the conversion effort (either develop a new nonGovernment standard or revise an existing non-Government standard), and PAPO's proposed level of involvement. Conversion efforts follow these plans, which are updated continuously to reflect ongoing activities.

\subsection{DEVELOPMENT OF A SYSTEM TO MONITOR CONJERSION ACTIVITIES}

The status information in this report has been developed to monitor the conversion activities in progress. Information provided by representatives of the non-Government standards bodies on progress achieved with development of the NGS, is incorporated, and the current status of conversion is reflected by appropriate entries.

The status of conversion has five parts: Part $I$ reflects the number and title of the standard recommended for conversion; Part II consists of the DOE standard status; Part III identifies the non-Government standards bodies, committees, and contacts; Part IV is the nonGovernment standards number, title, and status; and Part $V$ is the chronology of the project. 
4

\section{STATUS OF CONVERSION OF DOE STANDARDS}

TO NON-GOVERNMENT STANDARDS

To date, thirty DOE-NE standards have been converted to non-Government standards. There are nineteen standards presently in the conversion process. The status of these 19 conversion activities is summarized in the following pages. 
CONVERSION OF DOE STANDARDS TO NON-GOVERNMENT STANDARDS (NGS) JULY 1992

LEGEND for chronology of Project: (1) Identified for conversion, (2) DOE approval to submit to a Non-Government Standards Body (NGSB) for consideration, (3) Information package prepared, (4) to NGSB, (5) NGS begun, (6) NGS published, (7) NE Standard and NGS compared to determine degree of conversion and status of NE Standard.

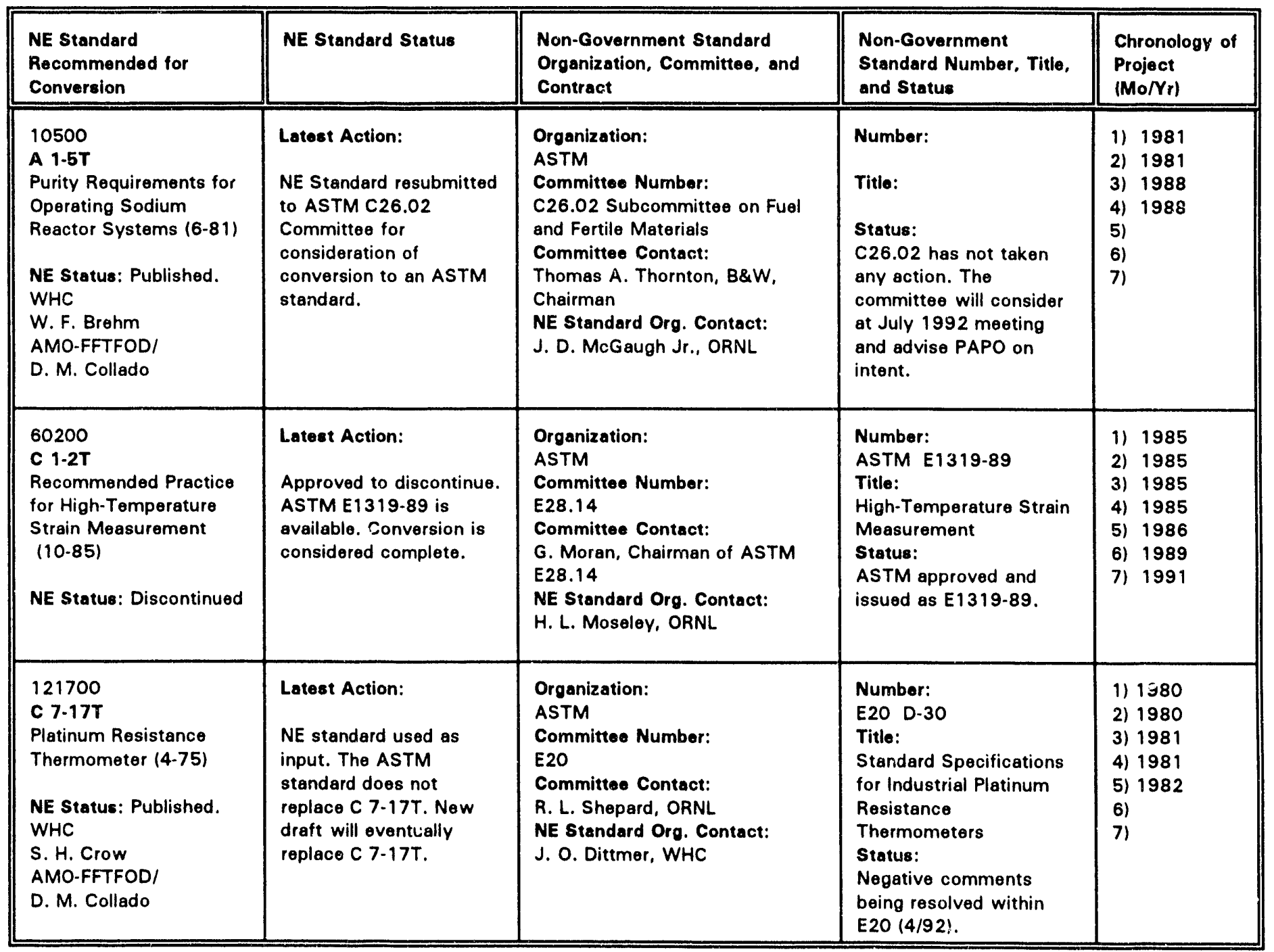


LEGEND for chronology of Project: (1) Identified for conversion, (2) DOE approval to submit to a Non-Government Standards Body (NGSB) for consideration, (3) Information package prepared, (4) to NGSB, (5) NGS begun, (6) NGS published, (7) NE Standard and NGS compared to determine degree of conversion and status of NE Standard.

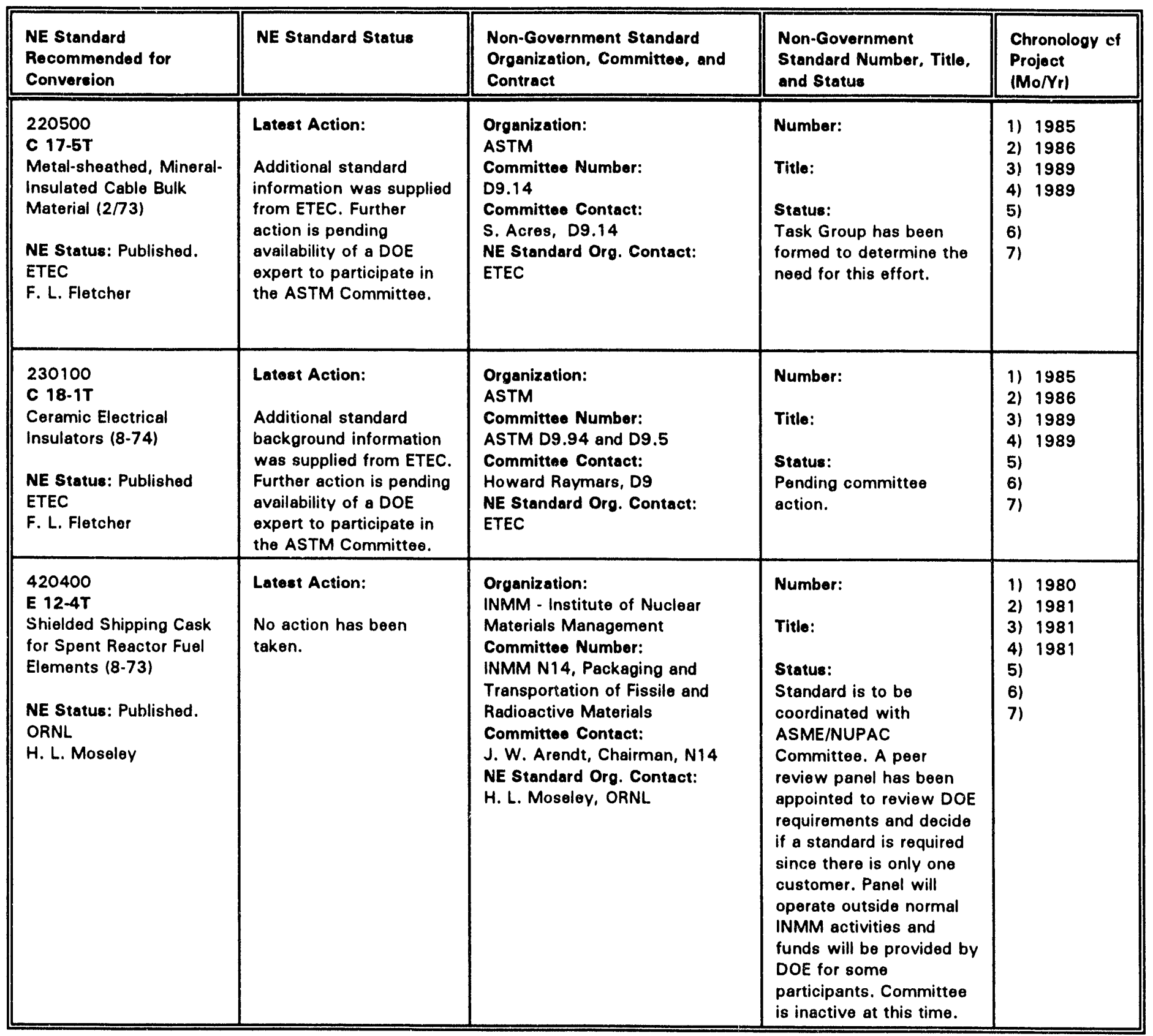


CONVERSION OF DOE STANDARDS TO NON-GOVERNMENT STANDARDS (NGS) JULY 1992

LEGEND for chronology of Project: (1) Identified for conversion, (2) DOE approval te submit to a Non-Government Standards Body (NGSB) for consideration, (3) Information package prepared, (4) to NGSB, (5) NGS begun, (0) NGS published, (7) NE Standerd and NGS compared to determine degree of conversion and status of NE Standard.

\begin{tabular}{|c|c|c|c|c|}
\hline $\begin{array}{l}\text { NE Standard } \\
\text { Recommended for } \\
\text { Convereion }\end{array}$ & NE Standard Status & $\begin{array}{l}\text { Non-Government Standard } \\
\text { Organization, Committee, and } \\
\text { Contract }\end{array}$ & $\begin{array}{l}\text { Non-Government } \\
\text { Standard Number, Title, } \\
\text { and Status }\end{array}$ & $\begin{array}{l}\text { Chronology of } \\
\text { Projoct (Mo/Nr) }\end{array}$ \\
\hline $\begin{array}{l}420500 \\
\text { E } 12-5 T \\
\text { Operating Manuals for } \\
\text { Fuel Shipping containers } \\
(1-75) \\
\text { NE Status: } \\
\text { Discontinued. }\end{array}$ & $\begin{array}{l}\text { Latest Action: } \\
\text { NE Standard has been } \\
\text { approved for } \\
\text { discontinuance. ANSI } \\
\text { N14.29-1988 is } \\
\text { available. Conversion is } \\
\text { considered complete. }\end{array}$ & $\begin{array}{l}\text { Organization: } \\
\text { INMM } \\
\text { Committee Number: } \\
\text { N14, Packaging and } \\
\text { Transportation of Fissile and } \\
\text { Radioactive Material } \\
\text { Committee Contact: } \\
\text { J. W. Arendt, Chairman N14 } \\
\text { J. W. Arendt, Coordinator } \\
\text { R. T. Waite, Chairman, N14.29 } \\
\text { NE Standard Org. Contact: } \\
\text { H. L. Moseley, ORNL }\end{array}$ & $\begin{array}{l}\text { Number: } \\
\text { ANSI/INMM, N14.29 } \\
\text { (N679) } \\
\text { Title: } \\
\text { Guide for Writing } \\
\text { Operating Manuals for } \\
\text { Radioactive Materials } \\
\text { Packaging } \\
\text { Status: } \\
\text { ANSI N14.29-1988 has } \\
\text { been published }(6 / 90)\end{array}$ & $\begin{array}{l}\text { 1) } 1976 \\
\text { 2) } 1981 \\
\text { 3) } 1981 \\
\text { 4) } 1981 \\
\text { 5) } 1980 \\
\text { 6) } 1990 \\
\text { 7) } 1991\end{array}$ \\
\hline $\begin{array}{l}420700 \\
\text { E 12-7T } \\
\text { Inspection and } \\
\text { Preventive Maintenance } \\
\text { of Fuel Shipping } \\
\text { Containers (1-75) } \\
\text { NE Status: Published. } \\
\text { ORNL } \\
\text { H. L. Moseley }\end{array}$ & $\begin{array}{l}\text { Latest Action: } \\
\text { No activity has been } \\
\text { undertaken. }\end{array}$ & $\begin{array}{l}\text { Organization: } \\
\text { INMM } \\
\text { Committee Number: } \\
\text { INMM N14, Packaging and } \\
\text { Transportation of Fissile and } \\
\text { Radioactive Materials } \\
\text { Committee Contact: } \\
\text { J. W. Arendt, Chairman, N14; } \\
\text { J. W. Arendt, Coordinator } \\
\text { NE Standard Org. Contact: } \\
\text { H. L. Moseley, ORNL }\end{array}$ & $\begin{array}{l}\text { Number: } \\
\text { ANSI/INMM N14.26 } \\
\text { Title: } \\
\text { Guidance on Quality } \\
\text { Control Activities as } \\
\text { They Relate to the } \\
\text { Inspection, Preventive } \\
\text { Maintenance, and Post- } \\
\text { Incident Testing of } \\
\text { Packages Used for the } \\
\text { Shipment of Rariioective } \\
\text { Material. } \\
\text { Status: } \\
\text { Committee has been } \\
\text { formed. Scopu is to be } \\
\text { prepared. }\end{array}$ & $\begin{array}{l}\text { 1) } 1980 \\
\text { 2) } 1981 \\
\text { 3) } 1981 \\
\text { 4) } 1981 \\
\text { 5) } \\
\text { 6) } \\
\text { 7) }\end{array}$ \\
\hline $\begin{array}{l}430200 \\
\text { E 13-2T } \\
\text { Ceramic Grade Uranium } \\
\text { Dioxide }(1 / 84) \\
\text { NE Status: Published. } \\
\text { WHC } \\
\text { J. O. Dittmer } \\
\text { AMO-FFTFOD/ } \\
\text { D. M. Collado }\end{array}$ & $\begin{array}{l}\text { Latest Action: } \\
\text { NE Standard has been } \\
\text { reviewed within WHC } \\
\text { against the revision to } \\
\text { the ASTM Standard. } \\
\text { Congnizant engineer } \\
\text { recommended retention } \\
\text { and inactivation of the } \\
\text { NE Standerd. }\end{array}$ & $\begin{array}{l}\text { Organization: } \\
\text { ASTM C26 Nuclear Fuel Cycle } \\
\text { Committee } \\
\text { Committee Number: } \\
\text { ASTM C26.02 Subcommittee on } \\
\text { Fuel and Fertile Materials } \\
\text { Committee Contact: } \\
\text { Thomas A. Thornton, B\&W, } \\
\text { Chairman, ASTM C25.02. } \\
\text { NE Standard Org. Contact. } \\
\text { H. L. Moseley, ORNL }\end{array}$ & $\begin{array}{l}\text { Number: } \\
\text { ASTM C753 } \\
\text { Title: } \\
\text { Standard Specification } \\
\text { for Nuclear-Grade, } \\
\text { Sinterable Uranium } \\
\text { Dioxide Powder. } \\
\text { Status: } \\
\text { The ASTM Standard has } \\
\text { been revised and } \\
\text { received C26 and ASTM } \\
\text { approval. Revised ASTM } \\
\text { standard does not } \\
\text { address all requirements } \\
\text { of the NE E 13-2T } \\
\text { standard. }\end{array}$ & $\begin{array}{l}\text { 1) } 1986 \\
\text { 2) } 1986 \\
\text { 3) } 1986 \\
\text { 4) } 1987 \\
\text { 5) } 1987 \\
\text { 6) } 1988 \\
\text { 7) }\end{array}$ \\
\hline
\end{tabular}




\section{CONVERSION OF DOE STANDARDS TO NON-GOVERNMENT STANDARDS (NGS) JULY 1992}

LEGEND for chronology of Project: (1) Identified for conversion, (2) DOE approval to submit to a Non-Government Standards Body (NGSB) for consideration, (3) Information package prepared, (4) to NGSB, (5) NGS begun, (6) NGS published, (7) NE Standard and NGS compared to determine degree of conversion and status of NE Standard.

\begin{tabular}{|c|c|c|c|c|}
\hline $\begin{array}{l}\text { NE Standard } \\
\text { Recommended for } \\
\text { Conversion }\end{array}$ & NE Standard Status & $\begin{array}{l}\text { Non-Government Standard } \\
\text { Organization, Committee, and } \\
\text { Contract }\end{array}$ & $\begin{array}{l}\text { Non-Government } \\
\text { Standard Number, Title, } \\
\text { and Status }\end{array}$ & $\begin{array}{l}\text { Chronology of } \\
\text { Project } \\
\text { (Mo/Yr) }\end{array}$ \\
\hline $\begin{array}{l}560200 \\
\text { F } 6-2 T \\
\text { Welding of Reactor Core } \\
\text { Components and Test } \\
\text { Assemblies (7-73) } \\
\text { NE Status: Published. } \\
\text { WHC } \\
\text { T. A. Delucchi } \\
\text { AMO-FFTFOD/ } \\
\text { D. M. Collado }\end{array}$ & $\begin{array}{l}\text { Latest Action: } \\
\text { No action pending } \\
\text { completion of the review } \\
\text { by AWS. }\end{array}$ & $\begin{array}{l}\text { Organization: } \\
\text { The American Welding Society is } \\
\text { reviewing the standard. } \\
\text { Committee Number: } \\
\text { Committee Contact: } \\
\text { H. Glenn Ziegenfuss, AWS } \\
\text { NE Standard Org. Contact: } \\
\text { H. L. Moseley, ORNL }\end{array}$ & $\begin{array}{l}\text { Number: } \\
\text { Title: } \\
\text { Status: } \\
\text { The Arnerican Welding } \\
\text { Society staff is } \\
\text { continuing to review the } \\
\text { standard with the } \\
\text { responsible Technical } \\
\text { Committee. }\end{array}$ & $\begin{array}{ll}\text { 1) } & 1985 \\
\text { 2) } & 1985 \\
\text { 3) } & 1986 \\
\text { 4) } & 1986-87 \\
\text { 5) } & \\
\text { 6) } & \\
\text { 7) } & \end{array}$ \\
\hline
\end{tabular}


CONVERSION OF DOE STANDARDS TO NON-GOVERNMENT STANDARDS (NGS) JULY 1992

LEGEND for chronology of Project: (1) Identified for conversion, (2) DOE approval to submit to a Non-Government Standards Body (NGSB) for consideration, (3) Information package prepared, (4) to NGSB, (5) NGS begun, (6) NGS published, (7) NE Standard and NGS compared to determine degree of conversion and status of NE Standard.

\begin{tabular}{|c|c|c|c|c|}
\hline $\begin{array}{l}580600 \\
\text { F } 8-6 T \\
\text { Hoisting and Rigging of } \\
\text { Critical Components and } \\
\text { Related Equipment } \\
(11-85) \\
\text { NE Status: Published. } \\
\text { Limited coordination } \\
\text { standard. } \\
\text { PM/ORNL } \\
\text { H. L. Moseley } \\
\text { EM-421 } \\
\text { J. W. Wagoner }\end{array}$ & $\begin{array}{l}\text { Latest Action: } \\
\text { Information package } \\
\text { completed and } \\
\text { forwarded to ASME for } \\
\text { consideration as basis of } \\
\text { new ASME standard. }\end{array}$ & $\begin{array}{l}\text { Organization: } \\
\text { ASME } \\
\text { Committee Number: } \\
\text { Main Committee on Cranes for } \\
\text { Nuclear Facilities } \\
\text { Committee Contact: } \\
\text { W. Berger, ASME } \\
\text { NE Standerd Org. Contact: } \\
\text { H. L. Moselev, ORNL }\end{array}$ & $\begin{array}{l}\text { Number: } \\
\text { Titlo: } \\
\text { Status: } \\
\text { Certain portions of the } \\
\text { standard will be } \\
\text { addressed in the future. } \\
\text { Committee is not active, } \\
\text { therefore conversion } \\
\text { activily will be extended. }\end{array}$ & $\begin{array}{ll}\text { 1) } & 1988 \\
\text { 2) } & 1988 \\
\text { 3) } & 1988 \\
\text { 4) } & 1988 \\
\text { 5) } & \\
\text { 6) } & \\
\text { 7) } & \end{array}$ \\
\hline
\end{tabular}




\section{CONVERSION OF DOE STANDARDS TO NON-GOVERNMENT STANDARDS (HGS) JULY 1992}

LEGEND for chronology of Project: (1) Identified for conversion, (2) DOE approval to submit to a Non-Government Standards Body (NGSB) for consideration, (3) Information packege prepared, (4) to NGSB, (5) NGS begun, (6) NGS published, (7) NE Standard and NGS compered to determine degree of conversion and status of NE Standard.

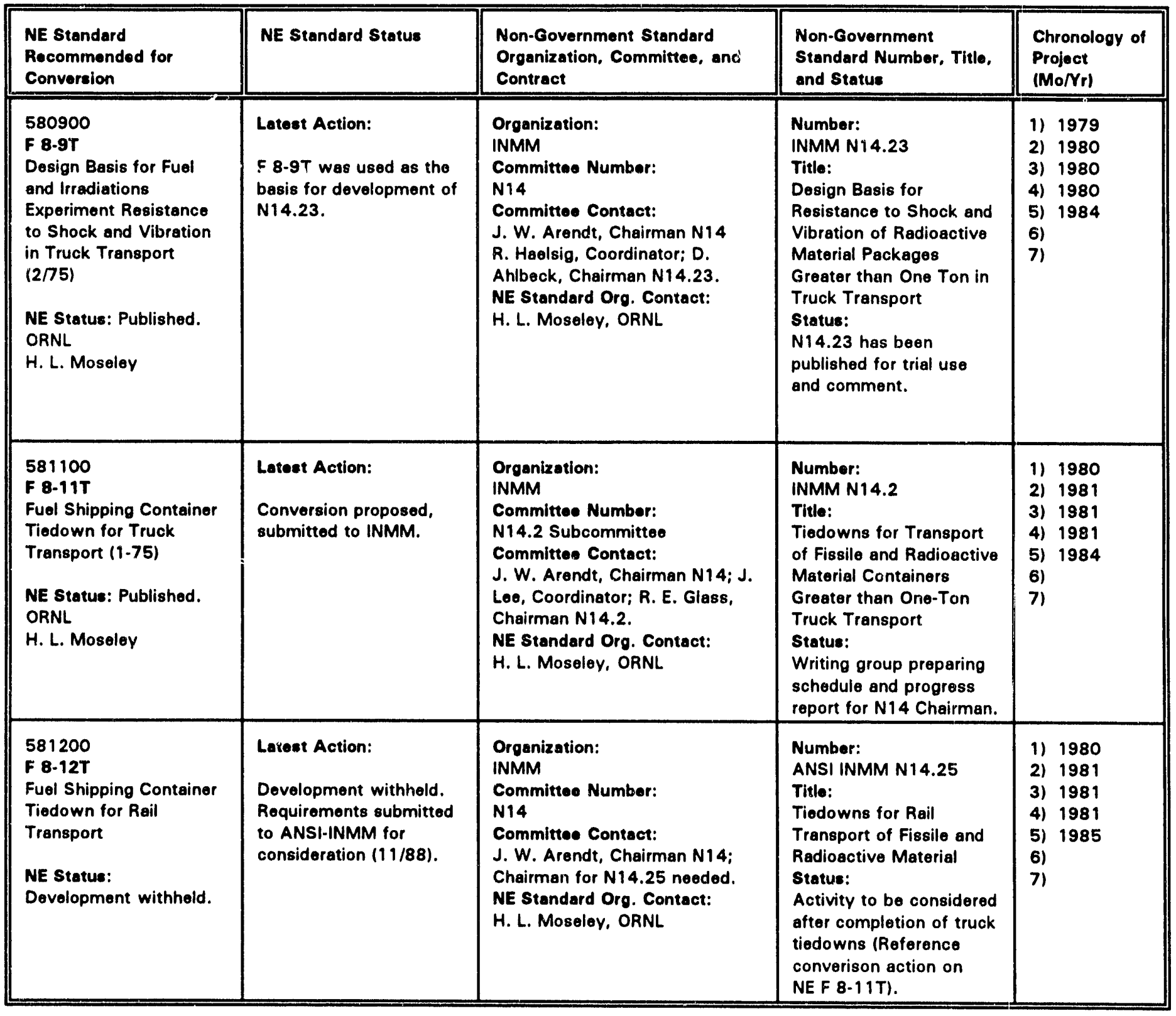


LEGEND for chronology of Project: (1) Identified for conversion, (2) DOE approval to submit to a Non-Government Standards Body (NGSB) for consideration, (3) Information package prepared, (4) to NGSB, (5) NGS begun, (6) NGS published, (7) NE Standard and NGS compared to determine degree of conversion and status of NE Standard.

\begin{tabular}{|c|c|c|c|c|}
\hline $\begin{array}{l}\text { NE Standard } \\
\text { Recommended for } \\
\text { Conversion }\end{array}$ & NE Standard Status & $\begin{array}{l}\text { Non-Government Standard } \\
\text { Organization, Committeo, and } \\
\text { Contract }\end{array}$ & $\begin{array}{l}\text { Non-Government } \\
\text { Standard Number, Title, } \\
\text { and Status }\end{array}$ & $\begin{array}{l}\text { Chronology of } \\
\text { Projoct } \\
\text { (Mo/Yr) }\end{array}$ \\
\hline $\begin{array}{l}770400 \\
\text { M } 12-4 T \\
\text { Reflective Insulation } \\
(1 / 72) \\
\text { NE Status: Published. } \\
\text { ORNL } \\
\text { D. Yarbrough } \\
\text { AMO-FFTFODI } \\
\text { D. M. Collado }\end{array}$ & $\begin{array}{l}\text { Latest Action: } \\
\text { Pending issue of ASTM } \\
\text { C667. }\end{array}$ & $\begin{array}{l}\text { Organization: } \\
\text { ASTM } \\
\text { Committee Number: } \\
\text { ASTM C16.21 Reflective } \\
\text { Insulation } \\
\text { Committee Contact: } \\
\text { F. J. Powell, chairman; Andra } \\
\text { Desjarais, Secretary; D. } \\
\text { Yarbrough, Chairman C16.21; } \\
\text { George Barnett, ASTM C667. } \\
\text { NE Standard Org. Contact: } \\
\text { David Yarbrough, ORNL }\end{array}$ & $\begin{array}{l}\text { Number: } \\
\text { C335-84, c1033-85 } \\
\text { Title: } \\
\text { Standard Test Method } \\
\text { for Steady-State Heat } \\
\text { Transfer Properties of } \\
\text { Horizontal Pipe } \\
\text { Insulation. (C335-84) } \\
\text { Standard Test Method } \\
\text { for Steady-State Heat } \\
\text { Transfer Properties of } \\
\text { Pipe Insulation Installed } \\
\text { Vertically. (C1033-85) } \\
\text { Statue: } \\
\text { C335-84, C691-84, and } \\
\text { C1033-85 have been } \\
\text { issued. C691-84 will be } \\
\text { withdrawn since the } \\
\text { latest revision of } \\
\text { C335-84 covers all } \\
\text { information now in } \\
\text { C691. A new draft of } \\
\text { C667 that covers } \\
\text { material properties was } \\
\text { accepted by C16.21. } \\
\text { The C667-89 draft } \\
\text { received negative votes } \\
\text { on the C16 committee } \\
\text { ballot. Negative votes } \\
\text { are being resolved. }\end{array}$ & $\begin{array}{ll}\text { 1) } & 1979 \\
\text { 2) } & 1979 \\
\text { 3) } & 1979 \\
\text { 4) } & 1979 \\
\text { 5) } & 1980 \\
\text { 6) } & \\
\text { 7) } & \end{array}$ \\
\hline $\begin{array}{l}790100 \\
\text { M 14-1T } \\
\text { Sodium Cover Gas - } \\
\text { Purchase Specifications } \\
\text { (7/72) } \\
\text { NE Status: Published. } \\
\text { WHC } \\
\text { W. J. Schuck } \\
\text { AM)-FFTFOD } \\
\text { D. M. Collado }\end{array}$ & $\begin{array}{l}\text { Lateet Action: } \\
\text { Review of M 14-1 and } \\
\text { ASTM C } 1143 \text { for degree } \\
\text { of conversion has been } \\
\text { completed. } \\
\text { All of the NE Standard } \\
\text { requirements are not } \\
\text { covered in the ASTM } \\
\text { Standard. DOE project } \\
\text { authorization will be } \\
\text { initiated to determine the } \\
\text { next action. }\end{array}$ & $\begin{array}{l}\text { Organization: } \\
\text { ASTM } \\
\text { Committoe Number: } \\
\text { ASTM C26.02 } \\
\text { Committee Contact: } \\
\text { Thomas A. Thornton, B\&W } \\
\text { NE Standard Org. Contact: } \\
\text { H. L. Moseley, ORNL }\end{array}$ & $\begin{array}{l}\text { Number: } \\
\text { ASTM C1143 } \\
\text { Title: } \\
\text { Standard Specification } \\
\text { for Helium for Use as a } \\
\text { Cover Gas for Liquid } \\
\text { Sodium. } \\
\text { Status: } \\
\text { New ASTM Standard has } \\
\text { been published. }\end{array}$ & $\begin{array}{ll}\text { 1) } & 1979 \\
\text { 2) } & 1984 \\
\text { 3) } & 1984 \\
\text { 4) } & 1984 \\
\text { 5) } & 1987 \\
\text { 6) } & 1989 \\
\text { 7) } & 1991\end{array}$ \\
\hline
\end{tabular}


CONVERSION OF DOE STANDARDS TO NON-GOVERNMENT STANDARDS INGS) JULY 1992

LEGEND for chronology of Project: (1) Identified for conversion, (2) DOE approval to submit to a Non-Government Standards Body (NGSB) for consideration, (3) Information package prepared, (4) to NGSB, (5) NGS begun, (6) NGS published, (7) NE Standard and NGS compared to determine degree of conversion and status of NE Standard.

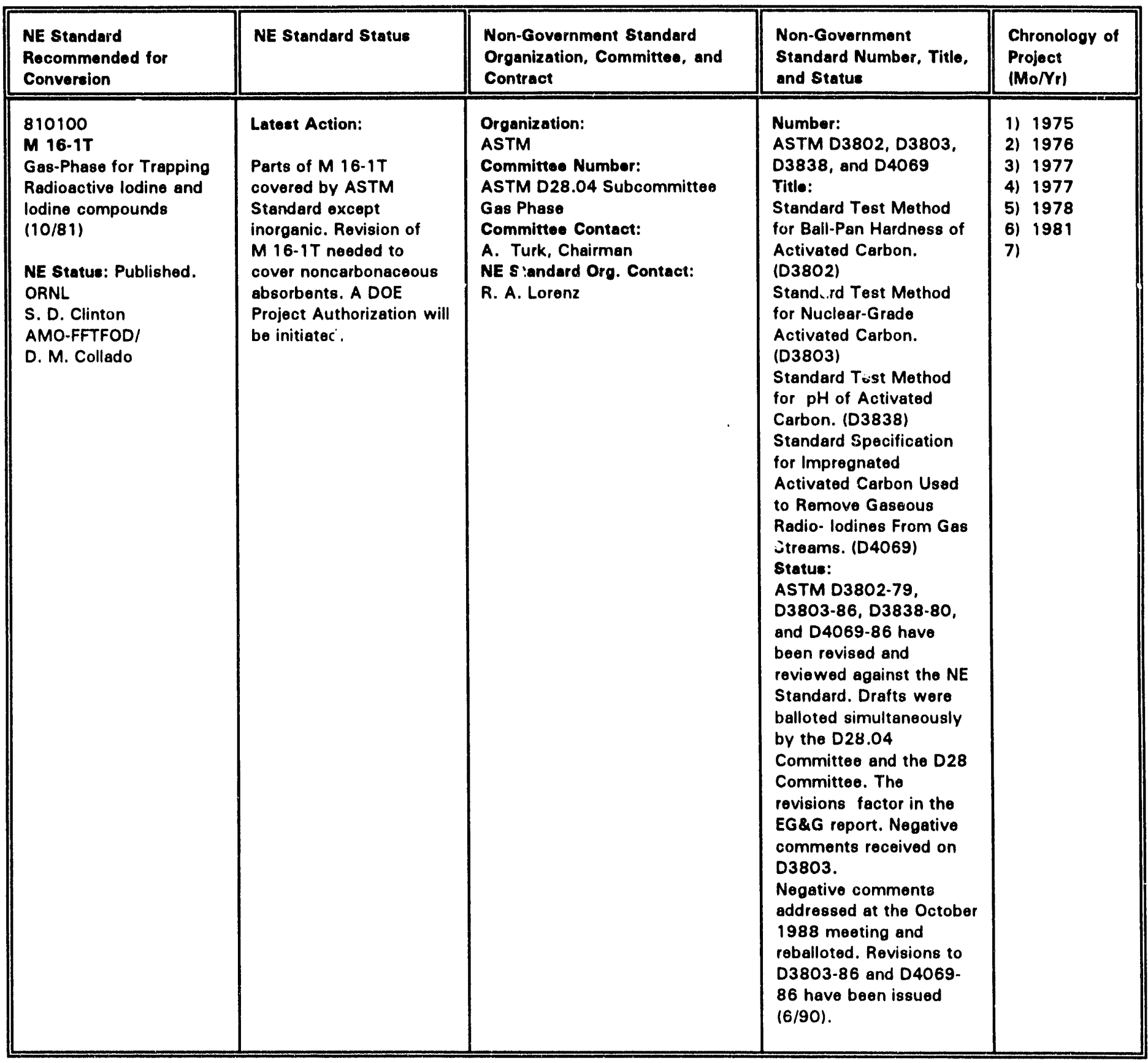




\section{Internal Distribution}

1.

B. B. Bevard

2. R. S. Booth

3. J. M. Corum

4. T. W. Horning

$5-$

11. S. D. Jennings

12. J. E Jones Jr.

13. W. R. Laing

14. H. L. Moseley

15. D. L. Moses

$16-$

21
F. C. Olden
22.
K. H. Poteet
23. R. L. Shepard
24. D. J. Spellman
25. J. D. White
26. D. L. Williams, Jr.
27. ORNL Central Research Library
28. Patent Office
29-
30. Laboratory Records Dept.
31. Laboratory Records - RC

\section{External Distribution}

32. L. E. Alsager, Consultant, 7420 Zenith Court, Falls Church, VA 22042

33. H. Alter, Office of Technical Support, Department of Energy, NE-462, Washington, DC 20585

34. V. G. Aquino, Quality Assurance, Argonne National Laboratory, P.O. Box 2528, Idaho Falls, ID 83403-2528

35. W. S. Barak, EBR-II, Argonne National Laboratory - West, P. O. Box 2528, Idaho Falls, ID 83401

36. J. G. Barr, Jr., San Francisco Field Office, Department of Energy, 1333 Broadway, Oakland, CA 94612

37. S.K. Beamer, Librarian, Energy Technology Engineering Center, Rockwell International, P. O. Box 1449, Canoga Park, CA 91304

38. R. T. Bell, Deputy Assistant Secretary for Health, Department of Energy, EH-411 GTN, Washington, DC 20585

39. J. W. Bennett, Director, Operations and Facility Reliability, Department of Energy UEA, NE-33 GTN, Washington, DC 20585

40. C. C. Bigelow, Office of Defense Energy Projects, Department of Energy, NE-52, Washington, DC 20585

41. E. F. Bjoro, Office of Nuclear Energy, Department of Energy, NE-84, Washington, DC 20585

42. L. F. Blankner, Oak Ridge Field Office, Department of Energy, P. O. Box 2001, Oak Ridge, TN 37831

43. K. C. Bowles, Sandvik Special Metals, P. O. Box 6027, Kennewick, WA 99336

44. H. R. Brager, Westinghouse Hanford Company, Building 326 - W/A-64, P. O. Box 1970, Richland, WA 99352

45. N. W. Brown - M/C S06, Space Nuclear Engineering and Technology, General Electric Company, 6835 Via Del Oro, Box 530954, San Jose, CA 95153-5354

46. W. S. Brown, Technical Assistant, Nuclear Technology Division, Westinghouse Electric Corporation, P. O. Box 355, Pittsburgh, PA 15230

47. W. J. Brynda, Associated Universities, Inc., Brookhaven National Laboratory, Building 703, Nuclear Standards Group, Upton Long Island, NY 11973 
48. M. A. Butler, Brookhaven Area Office, Department of Energy, Building 464, Upton Long Island, NY 11973

49. S. P. Carfagno, Division of Arvin/Calspan, Franklin Research Center, Valley Forge Corporate Center, 2600 Monroe Blvd., Norristown, PA 19403

50. A. P. Carmichael, Technical Library - 11A4, Carolina Power and Light Company, P. 0. Box 1551, Raleigh, NC 27602

51. W. E. Carnes, Risk and Policy Analysis Divisi'on, Department of Energy, NS-40 GTN, Washington, DC 20585

52. T. R. Colandrea, GA Technologies Inc., P.O. Box 85608, San Diego, CA 92138

53. R. Collins, Office of Proffessional and Technical Recruitment Training and Development, Department of Energy, AD-70 FORS, Washington, DC 20585

54. G. P. Connors, GEGA Corporation, P.O. Box 85608, 3550 General Atomics Court, San Diego, CA 92138-5608

55. L. R. Dahl, Space Nuclear Engineering and Technology, General Electric Company, P. O. Box 530954, San Jose, CA 95135-5354

56. K. S. Dawsor, NPR, EG\&G Idaho H. Q. Room 203, P.O. Box 1625, Idaho Falls, ID 83401

57. A. Delapaz, Nuclear Effects Laboratory, U.S. Army Missile Range, White Sands, NM 88002

58. Director, Oper. and Environ. Safety Div., CH, Department of Energy, 9800 South Cass Avenue, Argonne, IL 60439

59. P. J. Dirkmaat, Idaho Field Office, Department of Energy, 785 DOE Place, Idaho Falls, ID 83402

60. J. O. Dittmer, Westinghouse Hanford Company, P.O. Box 1970, MSIN-L4-34, Richland, WA 99352

61. D. E. Elias, Commonwealth Edison Company, 34 FN West, P. O. Box 767, Chicago, IL 60690

62. S. H. Evans, Semco Instruments, Inc., 11505 Vanowen Street, N. Hollywood, CA 91605

63. N. M. Ewbank, Nuclear Eng. - 278F2 OBA, The Detroit Edison Company, 2000 Second Avenue, Detroit, MI 48226

64. O. A. Farabee, Cheif, Reactor Programs, Department of Energy - AMO, P.O. Box 550, Richland, WA 99352

65. F. E. Faris, Suite 307, Crystal Plaza One, Interdevelopment, Inc., 2001 S. Jefferson Davis Highway \#307, Arlington, VA 22202-3603

66. FERMI 2 Power Plant, Detroit Edison Company, NOC Info. Ctr., - 160 NOC, 6400 North Dixie Highway, Newport MC 48166

67. S. M. Fingerman, Information Center, Teledyne Engineering Services, 130 Second Avenue, Waltham, MA 02254

68. M. J. Fox, Dynamics Corporation of America, Ellis and Watts, P.O. Box 44010, Cincinnati, OH 45227

69. E. O. Fromm, Quality Assurance Manager, Nuclear Services Group, Battelle Columbus Laboratories, 505 King Avenue, Columbus, OH 43201

70. G. Gears, Deputy Assistant Secretary for Facilities, Department of Energy, DP-62 GTN, Washington, DC 20585

71. R. R. Geoffrion, Associate Group Leader for Quality Assurance, MST - MS G755, Los Alamos National Laboratory, P. O. Box 1663, Los Alamos, NM 87545

72. N. Goldenberg, Office of Nuclear Energy, Department of Energy, NE-70/GTN, Washington, DC 20585 
73. M. F. Haughey, Nuclear Operations and Analysis Division, Department of Energy, NE72 GTN, Washington, DC 20585

74. R. P. Hearn, Argonne National Laboratory - West, P.O. Box 2528, Idaho Falls, ID 83401

75. J. L Hechmer, Nuclear Equipment Division, Babcock and Wilcox Company, 91 Sterling Avenue, Barberton, $\mathrm{OH} 44203$

76. G. A. Hellarid, Deputy Assistant Secretary for Export Assistance, IE-30 FORS, Washington, DC 20585

77. K. R. Hendricks, Commercial Insspection Service Inc., 156 W. Provindencia Avenue, Burbank, CA 91502

78. E. Holby, Fluor Engineers Inc., 200 W. Monroe, Chicago, IL 60606

79. F. D. Hutchinson, Gibbs and Hill, Inc., 11 Penn Plaza, New York, NY 10001

80. D. A. Hutson, Space Nuclear Engineering and Technology, General Electric Company, P. O. Box 530954, San Jose, CA 95153-5354

81. J. W. Jacox, Partners in Technology, Jacox Associates, P. O. Box 29720, Columbus, $\mathrm{OH} 43201$

82. R. D. Jarrett, Office of University and Science Education Programs, ER-8.1 FORS, Washington, DC 20585

83. R. A. Jekel, Rockwell Hanford Operations, P. O. Box 800, Richland, WA 99352

84. W. D. Jensen, Director, Operational Safety Division, Idaho Field Office, Department of Energy, 785 DOE Place, Idaho Falls, ID 83402

85. R. I. Jetter, Manager Engineering, Energy Technology Engineering Center, P.O. Box 1449, Canoga Park, CA 91304

86. R. H. Johnson, Technical Library - UPGD, Babcock and Wilcox Company, P. O. Box 1260, Lynchburg, VA 24505

87. R. J. Kennedy, Nuclear Operations Support, Boston Edison Company, 25 Braintree Hill Office Park, Braintree, MA 02184

88. N. A. Kershaw, Administrative Engineer, Commonwealth Edison Company, 34-FNE, P. O. Box 767, Chicago, IL 60690

89. T. Kirk, Huntington Alloys Inc., Huntington, WV 25720

90. J. Kirkland, Department of Microforms, Georgia Institute of Technology, Library, 225 North Ave. NW, Atlanta, GA 30302

91. F. W. Knight, Waste Technology Services Division, Westinghouse Electric Corporation, P. O. Box 10864, Pittsburgh, PA 15236

92. N. L. Knight, Technical Information Center, General Physics Corporation, 10650 Hickory Ridge Road, Columbia, MD 21044

93. G. W. Knowles, Associated Machine, 1600 Martin Avenue, Santa Clara, CA 95050

94. R. Kramer, Office of New Production Reactors, NP-2 FORS, Washington, DC 20585

95. S. A. Larkin, Technical Information Center, Stone and Webster Engineering Corp., 3 Executive Campus, P. O. Box 5200, Cherry Hill, NJ 08034

96. R. A. Larson, Library, Argonne National Laboratory, P. O. Box 2528, Idaho Falls, ID 83403

97. D. R. Lehman, Construction Management Support, Department of Energy, ER-65, Washington, DC 20585

98. G. Santos-Leon, Office of New Production Reactors, NP-64, Department of Energy, Forrestal Bldg., 1000 Independence Ave., SW, Washington, DC 20585

99. J. Ling, Nuclear Codes and Standards, American Society of Mechanical Engineers, 345 East 47th Street, New York, NY 10017

100. Herbert D. Link, 5 Grandview Avenue, Pittsburgh, PA 15211 
101. M. C. Ma, Librarian, Nuclear Services Corporation, Quadrex Corporation, 1700 Dell Avenue, Campbell, CA 95008

102. E. F. Mack, Extrusion Facility, Curtiss-Wright Corporation, 60 Grider Street, Buffalo, SY 14215

103. T. E. Magette, Office of Safety and Quality, NP-32 FORS, Washington, DC 20585

104. D. K. Magnus, Senior Technical Advisor, Uranium Enrichment, Department of Energy, NE-30/GTN, Washington, DC 20585

105. J. M. Marzec, Rocketdyne Division, MS HB03, Rockwell International, 6633 Canoga Avenue, Canoga Park, CA 91304

106. G. W. May, Project Engineer, Research and Engineering, Bechtel Group, Inc., P. O. Bux 3965, San Francisco, CA 94119

107. H. Mayer, Library - P3C, Public Service Electric and Gas Company, P. O. Box 570, Newark, NJ 07101

108. J. N. McBride, Standards Coordinator, EG\&G Idaho, Inc., P.O. Box 1625, Idaho Falls, ID 83415

109. M. J. McCabe, Deputy Assistant Secretary for Building Technolgoeis, CE-413 FORS, Washington, DC 20585

110. C. J. McKenna, ITT Grinnell Corporation, 260 West Exchange Street, Providence, RI 02901

111. W. N. McLean, Crane Company, 475 N. Gary Avenue, Carol Stream, IL 60188

112. P. J. Meadowcroft, Technical Service Supervisor, Atomic Energy of Canada Limited, Chalk River Nuclear Laboratories, Chalk River ONT KOJ 1 JO, Canada

113. S. Metta, Applied Engineering, 1525 Charleston Highway, Orangeburg, SC 29115

114. R. E. Monks, Quality Assurance Engineer, Assistant Manager for Energy and Conservation, Nevada Field Office-DOE, P. O. Box 14100, Las Vegas, NV 89114

115. M. Moon, Office of Public Affairs, PA-5 FORS, Washington, DC 20585

116. J. T. Mulvey, ARI Industries Inc., 381 ARI Court, Addison, IL 60107

117. J. O. Neff, U. S. Department of Energy, 505 King Avenue, Columbus, OH 43201

118. W. R. Nicolls, Bonney Forge Division, G\&W Manufacturing Company, P. O. Box 359, Allentown, PA 18105

119. J. H. Noble, Chief Mechanical Engineer, Thermal Power, Charles T. Main, Inc., Prudential Center - Southeast, Boston, MA 02199

120. D. R. Nordell, Sr., Monticello Nuclear Generating Plant, P.O. Box 600, Monticello, MN 55362

121. D. A. Ottaviani, Quality Assurance Division, Perini Corporation, 73 Mt. Wayte Avenue, Framingham, MA 01701

122. E. H. Palys, Quality Assurance Division, Argonne National Laboratory, 9700 South Cass Avenue, Bldg. 201 - M/S QAS, Argonne, IL 60439-4832

123. R. J. Paolino, NRC:R1 PSS;DETP, U.S. Nuclear Regulatory Commission, 631 Park Avenue, King of Prussia, PA 19406

124. N. T. Patel, ABWR Program, GE Nuclear Energy, M/C 712, 175 Curtner Avenue, San Jose, CA 95125

125. K. C. Pearson, American Society for Testing and Materials, 1916 Race Street, Philadelphia, PA 19103

126. R. E. Pegram, Durkee Testing Laboratories, 15700 Texaco Street, Paramount, CA 90723-3924

127. M. Petro, Advanced Systems Division, Mine Safety Appliances Company, RD. \#2, Evans City, PA 16033

128. G. C. Pratt, Quality Assurance, Stearns-Catalytic, P. O. Box 5888, Denver, CO 80217 
129. Ankur Purohit, Engineering Physics Division, Argonne National Laboratory, 9700 South Cass Avenue, Argonne, IL 60439

131. M. Regan-Haugh, Deputy Assistant Secretary for Facilities, Department of Energy, DP62 GTN, Washington, DC 20585

132. G. W. Roberts, Research and Development Division, Babcock and Wilcox Company, 1562 Beeson Street, P. O. Box 835, Alliance, OH 44601

133. B. J. Rock, Deputy Assistant Secretary for Civilian Reactor Development, Department of Energy, NE-46 GTN, Washington, DC 20585

134. E. C. Rodabaugh, E. C. Rodabaugh Associates, Inc., 4625 Cemetery Road, Hilliard, $\mathrm{OH}$ 43026

134-

143. L. E. Rogers, Manager, DOE Standards Program, Department of Energy, NE-72 GTN, Washington, DC 20585

144. R. Rollins, Savannah River Field Office - OQA, Department of Energy, P.O. Box A, Aiken, SC 29808

145. C. H. Schmitt, Deputy Director, Deputy Assistant Secretary for Naval Reactors, NE-60 NR, NC-2 Bldg., 2521 Jefferson Davis Hwy., Arlington,VA 22202

146. E. Schwartz, Nuclear Fuel Division, Westinghouse Electric Corporation, MMOB215, P.O. Box 3912, Pittsburgh, PA 15230

147. W. B. Seefeldt, Chemical Engineering Division, Argonne National Laboratory, 9700 South Cass Avenue, Argonne, IL 60439

148. J. M. Selby, Battelle Pacific-Northwest Laboratory, P.O. Box 999, Richland, WA 99352

149. R. J. Serbu, Office of Safety and Quality Assurance, Department of Energy, EH-30.1 GTN, Washington, DC 20585

150. P. G. Sewell, Deputy Assistant Secretary for UE, Department of Energy, NE-30 GTN, Washington, DC 20585

151. R. Shechtel, Budget and Administration Staff, Department of Energy, EH-10 FORS, Washingtion, DC 20585

152. D. L. Sherman, Information/Records Director, Library, American Nuclear Insurers, 270 Farmington Ave., Suite 245, Farmington, CT 06032

153. R. L. Simon, Reuter-Stokes, Inc., Edison Park, 8499 Darrow Rd., Twinsburg, OH 44087

154. B. K. Singh, Office of Nuclear Energy, Department of Energy, NE-74/GTN, Washington, DC 20585

155. K. P. Singh, Central Engineering Division, Joseph Oat Corporation, 2500 Broadway, P. O. Box 10, Camden, NJ 08104

156. S. M. Smith, Office of Organization, Resources and Facilities Management, Department of Energy, AD-152 FORS, Washington, DC 20585

157. R. C. Smyth, Office of Quality Assurance and Quality Control, Department of Energy, EM-23 TREV, Washington, DC 20585

158. F. L. Stegenga, Space Nuclear Engineering and Technology, General Electric Company, 6835 Via Del Oro, P.O. Box 530954, MS S-05, San Jose, CA 95153-5354

159. K. E. Stevens, Parnall Technical Library, Consumers Power Company, 1945 Parnall Road, Jackson, Ml 49201

160. M. Sturdivant, Performance Assessment Division, Department of Energy, NS-10 GTN, Washington, DC 20585

161. J. R. Sumpter, Nuclear Services Department, Houston Lighting and Power Company, P. O. Box 1700, Houston, TX 77001

162. J. Susnir, Holmes and Narver, Inc., P.O. Box 6240, Orange, CA 92667-1240

163. K. .. Taber, Catalytic Inc., 1500 Market Street, Philadelphia, PA 19102 
164. M. L. Thompson, Space Nuclear Engineering and Technology, General Electric Company, 6835 Via Del Oro, P.O. Box 530954, San Jose, CA 95135-5354

165. Len Tiemann, Space Nuclear Engineering and Technology, MC S-05, General Electric Company, 6835 Via Del Oro, P.O. Box 530954, San Jose, CA $95135-5354$

166. H. K. Todosow, Nuclear Safety Library, Brookhaven National Laboratory, Building 130, Upton, NY 11973

167. T. N. Troy, Office Product Standards Policy, National Bureau of Standards, Technology Building - Room B166, Washington, DC 20234

168. D. H. Turno, Senior Supervisor, Technical Information Service, Westinghouse Savannah River Company, Savannah River Laboratory, Aiken, SC 29808

169 -

179. U.S. Nuclear Regulatory Commission, Document Control Desk, RIDS Code DOEX, Washington, DC 20555

180. D. M. VanSteinburg, Office of Professional and Technical Recruitment Training and Development, Department of Energy, AD-70 FORS, Washington, DC 20585

181. W. C. Vilona, Westinghouse Bettis Atomic Power Laboratory, G Building, 62JJ, P. 0. Box 79, West Mifflin, PA 15122-0079

182. F. J. Volpe, Office of Nuclear Energy, Department of Energy, NE-73/GTN, Washington, D.C. 20585

183. P. D. Weaver, Office of Policy, Department of Energy, PR-121 FORS, Washington, DC 20585

184. M. D. Weber, Manager, Standards Department, American Nuclear Society, 555 North Kensington Avenue, La Grange Park, IL 60525

185. A. White, Assistant Secretary for Fossil Energy, Department of Energy, FE- FORS, Washington, DC 20585

186. A. G. Wilkinson, Advanced Energy Systems Division, Westinghouse Electric Corporation, P.O. Box 158, Madison, PA 15663

187. R. Wilson, Office of Organization, Resources and Facilities Management, AD-131 FORS, Washington, DC 20585

188. D. M. Wirth, Library, Gilbert Associates, Inc., P.O. Box 1498, Reading, PA 19603

189. M. E. Wrenn, Radiobiology Department, University of Utah, Building 351, East 2nd South, Salt Lake City, UT 84112

190. F. B. Young, Eastern Stainless Steel Company, P. O. Box 1975, Baltimore, MD 21203

191. H. G. Ziegenfuss, Technical Director, Technical Department, American Welding Society, P.O. Box 351040, Miami, FL 33135

192. S. Zobel, Deputy Assistant Secretary for Health, Department of Energy, EH-40 GTN, Washington, DC 20585

193-

194. Office of Scientific and Technical Information, P. O. Box 62, Oak Ridge, TN 37830 

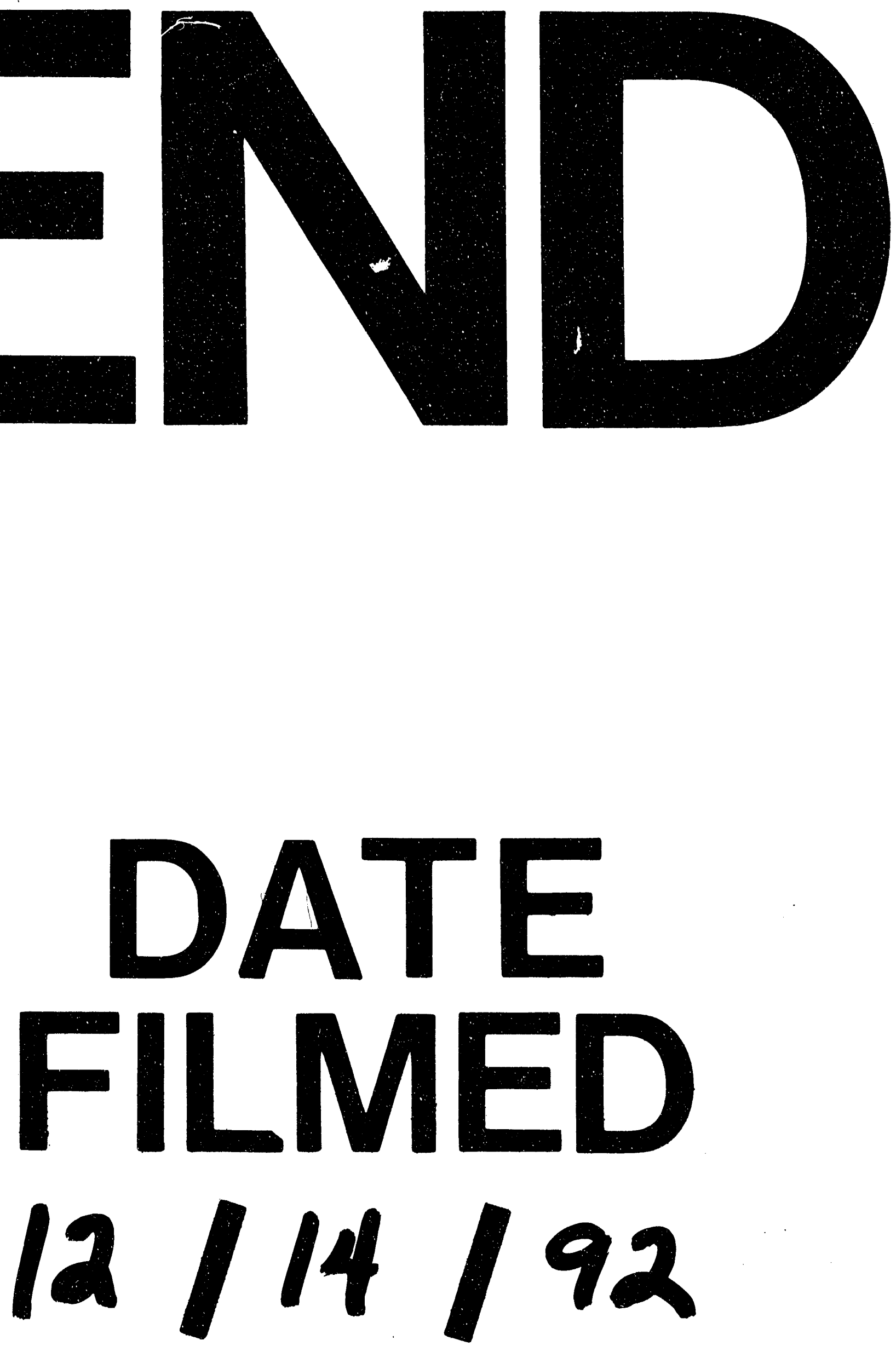
